\title{
mHealth Mindfulness Intervention for Women with Moderate-to-Moderately-Severe Antenatal Depressive Symptoms: a Pilot Study Within an Integrated Health Care System
}

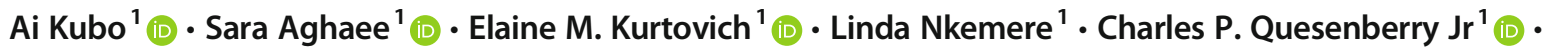 \\ MegAnn K. McGinnis ${ }^{1} \cdot$ Lyndsay A. Avalos $^{1}$ (D)
}

Accepted: 22 February 2021 / Published online: 11 March 2021

(C) The Author(s), under exclusive licence to Springer Science+Business Media, LLC, part of Springer Nature 2021

\begin{abstract}
Objectives Traditional mindfulness-based interventions have been shown to reduce depression symptoms in pregnant women, although in-person classes may pose significant accessibility barriers, particularly during the COVID-19 pandemic. Mobile technology offers greater convenience, but little is known regarding the efficacy of self-paced, mobile-delivered (mHealth) mindfulness interventions in this population. This study tested the feasibility and acceptability of offering such an intervention for pregnant women with moderate-to-moderately-severe depression symptoms.

Methods We conducted a single-arm trial within Kaiser Permanente Northern California (KPNC). Participants were identified through KPNC's universal perinatal depression screening program. Eligible participants included English-speaking pregnant women ( $<28$ weeks of gestation) with moderate-to-moderately-severe depressive symptoms without a regular (<3 times/week) mindfulness/meditation practice. Participants were asked to follow a self-paced, 6-week mindfulness meditation program using a mobile app, Headspace ${ }^{\mathrm{TM}}, 10-20 \mathrm{~min} /$ day. Outcome measures included feasibility, acceptability, and patient-reported outcomes (e.g., depression symptoms).

Results Of the 27 women enrolled, 20 (74\%) completed the study. Over half(55\%) of participants used the app $\geq 50 \%$ of the days during the 6-week intervention. Responses to the semi-structured interviews indicated that women appreciated the convenience of the intervention and the ability to engage without having to attend classes or arrange childcare. We observed significant improvements in pre-postintervention scores for depression symptoms, perceived stress, sleep disturbance, and mindfulness.

Conclusions Our study demonstrates the feasibility and acceptability of an mHealth mindfulness intervention for women with moderate-to-moderately-severe antenatal depression symptoms. The preliminary data further suggest that an efficacy trial is warranted.
\end{abstract}

Keywords Depression · Mindfulness · Mobile health · Pregnancy · Intervention · Prenatal $\cdot$ Mental health $\cdot$ Maternal health · Perinatal

Antenatal depression is a debilitating and costly mood disorder that affects approximately $12 \%$ of pregnant women (Gavin et al. 2005). It is the most prevalent complication of pregnancy and is associated with significant health consequences for mothers and children (Avalos et al. 2015; Badon et al. 2019; Grigoriadis et al. 2013; Szegda et al. 2014).

Ai Kubo

ai.kubo@kp.org

1 Kaiser Permanente Division of Research, 2000 Broadway, Oakland, CA 94612, USA
Though antenatal depression is the strongest predictor of postpartum depression, impacting up to $20 \%$ of postpartum women (Gavin et al. 2005; Gaynes et al. 2005), half of all women with antenatal depression do not receive treatment (Ko et al. 2012).

There are several barriers to current treatment options for women with antenatal depression. First, citing concerns over the safety of antidepressant medication use during pregnancy, most pregnant women (83-95\%) prefer non-pharmaceutical treatments (Dimidjian and Goodman 2009, 2014). Second, while psychotherapy is an effective non-pharmacological treatment option, numerous barriers to receiving care exist, including the shortage of mental health care providers 
(Kaiser Family Foundation n.d.), limited access to care, financial constraints, and lack of time, transportation, and childcare (Goodman 2009). Further, the current COVID-19 pandemic is posing a significant impact on the mental health of pregnant and postpartum women: a recent study reported a near tripling of the rate of perinatal depression since the pandemic began (15\% pre-pandemic versus $41 \%$ ) (Davenport et al. 2020). While rates of perinatal depression have increased, access to in-person delivered health care is drastically diminished ( $\mathrm{Li}$ 2020; Panchal et al. 2020). Therefore, it is critical to identify safe, remotely-delivered, effective, and scalable intervention options for pregnant women with depression.

Mindfulness, a psychological process of bringing one's attention to the present moment (Creswell 2017; Kabat-Zinn 1990; Pagnini and Philips 2015), is an effective intervention for reducing symptoms of depression in many populations (Dimidjian et al. 2016; Guardino et al. 2014; Khoury et al. 2015; Lever Taylor et al. 2016; Vieten and Astin 2008). Despite the promising evidence of mindfulness-based interventions in reducing depression symptoms, logistical requirements of currently available mindfulness training such as mindfulness-based stress reduction (MBSR) and mindfulness-based cognitive therapy (MBCT) (Kabat-Zinn 1990; Segal et al. 2002) often include over $30 \mathrm{~h}$ of in-person instruction together with $45 \mathrm{~min}$ of home practice daily, which severely restricts its accessibility for pregnant women. Women of reproductive age are often too busy to attend regular in-person sessions, particularly if they have other children at home and/or a full-time job. In fact, a recent in-person mindfulness pilot study for pregnant women reported that the recruitment was challenging for these reasons and that a more practical intervention was needed (Guardino et al. 2014). Logistical barriers such as distance, lack of transportation, childcare, and scheduling conflicts have been reported as reasons for non-adherence or refusal to participate in behavioral intervention research studies (Eyles et al. 2015; Schapira et al. 2014; Wahbeh et al. 2014a; Wahbeh and Oken 2016). In addition, in-person classes are costly for the patients and the health care system and not readily available in rural areas, preventing wide dissemination.

The widespread use of smartphones has led to a proliferation of behavioral Internet-based or mobile application (mHealth) interventions that fit the lifestyles of individuals who are unable to attend in-person classes and offer potential for improving adherence and efficacy. Remotely delivered interventions are also urgently needed as underscored by the COVID-19 pandemic. Recent studies report that many Americans suffering from depression, stress, or anxiety prefer online mindfulness training over in-person sessions (Wahbeh and Oken 2016; Wahbeh et al. 2014a, b). A recent metaanalysis of online, webinar versions of traditional mindfulness-based intervention (MBI) (e.g., MBSR) has demonstrated significant beneficial impact on depression, anxiety, and well-being in various populations (Spijkerman et al. 2016). However, studies on Internet-based mindfulness interventions are extremely limited among perinatal women with depression symptoms, and accessibility challenges remain. While the introduction of technology for delivery of MBI is a major advancement, these studies use online versions of the traditional MBIs in a webinar format with a trained facilitator. Thus, participants are still required to $\log$ on at a specified weekly time and there are the same extensive training durations (e.g., 30+ h total, $2.5 \mathrm{~h}$ a session) and homework (45 $\mathrm{min}$ /day) over 8 weeks, just as the in-person versions. A selfpaced, mHealth MBI with smaller dosage has high potential as a scalable and effective intervention with greater accessibility. Emerging studies have documented the efficacy of self-paced, smaller dosage digitally-delivered MBIs in reducing depression symptoms and stress in the general population (Economides et al. 2019; Ritvo et al. 2019; Yang et al. 2018) and among those with debilitating illnesses such as cancer (Bruggeman-Everts et al. 2017; Compen et al. 2018; Lengacher et al. 2018; Zernicke et al. 2014). However, little is known regarding the efficacy of self-paced mHealth mindfulness interventions among women with antenatal depression symptoms. As a first step, we conducted a feasibility study of such an intervention for pregnant women with moderate-tomoderately-severe depression symptoms within an integrated health care delivery system.

\section{Method}

\section{Participants}

We conducted a single-arm trial between March 2018 and May 2019. Women seeking antenatal care were recruited from 7 of the 44 Kaiser Permanente Northern California (KPNC) obstetrics and gynecology (OBGYN) clinics. Eligibility criteria included the following: age $\geq 18$ years, Patient Health Questionnare-9 (PHQ-9) score 10-19 (indicating moderateto-moderately-severe depression symptoms), <28 weeks of gestation, English-speaking, and access to a smartphone, tablet, and/or computer with Internet connection. Women who engaged in a regular mindfulness or meditation practice three or more times per week or were enrolled in a mindfulness program were ineligible.

A total of 531 women were identified to have a PHQ-9 score between 10 and 19 in the seven clinics. Among those, 275 were reached as potentially eligible participants $(n=259$ through EHR databases, $n=16$ from clinician referral) (Fig. 1). The primary reasons for exclusion were not meeting eligibility criteria $(n=114,41 \%)$ and declining to participate $(n=101$, $37 \%$ ). The most common reasons for ineligibility were gestational age $>28$ weeks, current pregnancy ended in miscarriage, PHQ-8 score out of the eligible range, and existing meditation 
Fig. 1 Overall flow of participants through the study

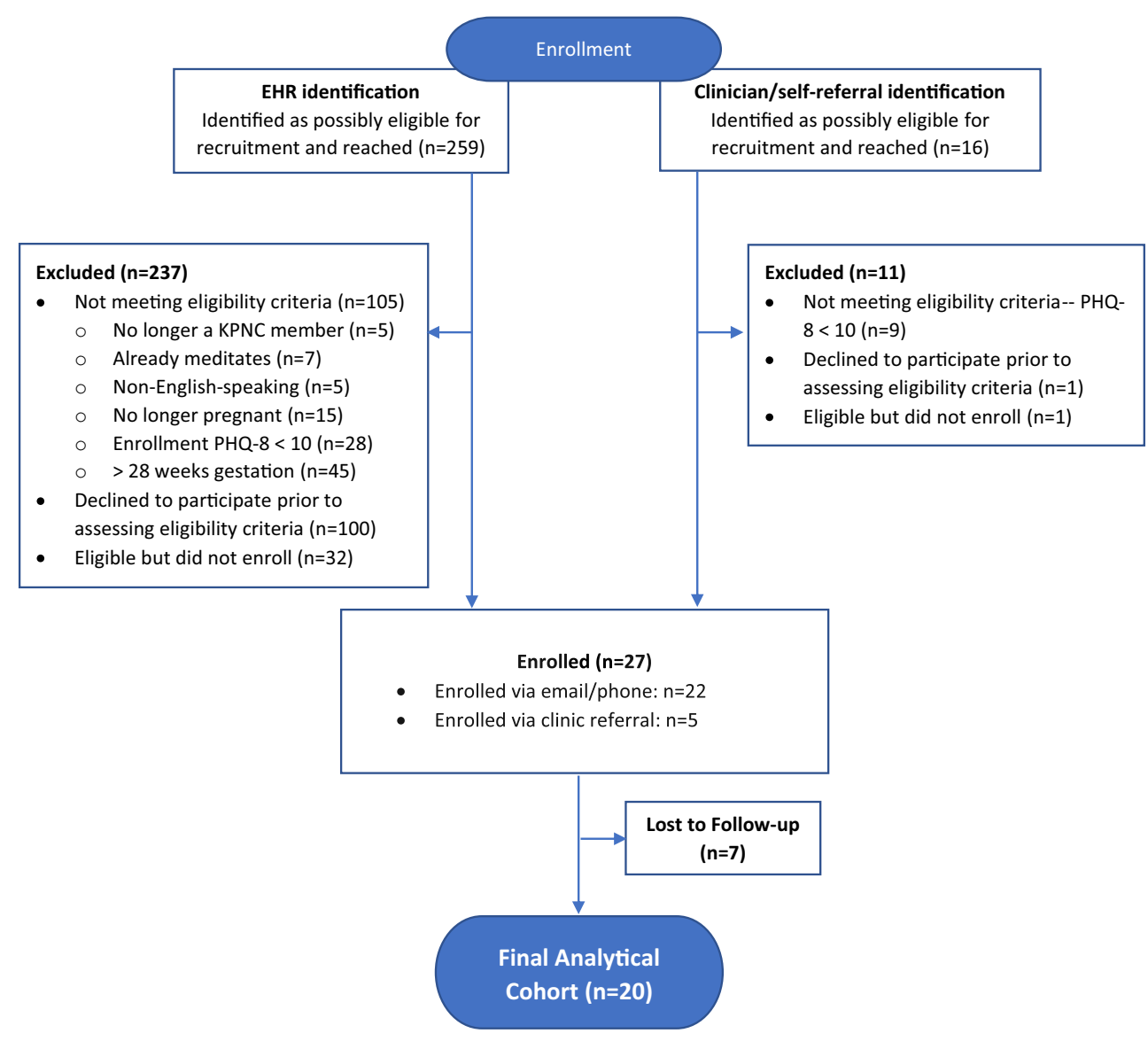

practice. The most common reasons for declining participation were being too busy and lack of interest. A total of 27 participants enrolled in the study.

\section{Procedures}

\section{Setting}

The study was conducted within KPNC, an integrated health care delivery system serving over 4.4 million racially- and socioeconomically-diverse members who are representative of the Northern California population (Gordon 2012, 2015). Standard antenatal care includes screening for depression at two points during pregnancy (first antenatal visit and glucola visit at 26-28 weeks of gestation) using the PHQ-9 (Kroenke et al. 2001).

\section{Recruitment}

Potential participants were identified using two strategies: (1) a universal perinatal depression screening PHQ-9 score of 1019 during pregnancy identified through the KPNC electronic health records (EHRs) and (2) clinician referral or self-referral from KPNC OBGYN clinical staff or study brochures. Potential participants were contacted about the study via email and phone by a research assistant and rescreened for depression symptoms using the PHQ-8 (see below for more information). Women who met all eligibility criteria and had a PHQ-8 score of 10-19 were enrolled. Informed consent was obtained from all individual participants included in the study. Participants then completed the baseline survey and received access to the mHealth mindfulness program (details below). Participants were asked to complete an online follow-up survey immediately after the intervention to assess patientreported outcomes, in addition to semi-structured interviews within 3 weeks after the intervention to assess acceptability of the intervention. Women who completed both baseline and 6week follow-up surveys received a \$25 gift card and an additional year-long subscription to the mindfulness app. The KPNC Institutional Review Board approved the study.

\section{Intervention}

The intervention used a commercially available mindfulness program, Headspace ${ }^{\mathrm{TM}}$, which provides self-paced, guided mindfulness meditations through a website or mobile application (iOS and Android). Women were asked to use the app for 10-20 min a day over the 6-week study period. Each participant was given a study-specific log-in ID and instructed to first complete the 30-day "Basics" course, which teaches users 
the basics of mindfulness meditation. After that, they could choose other 10- to 30-day courses that are condition- or situation-specific, such as "Pregnancy," "Anxiety," "Relationships," or "Sleep," or single meditation sessions for the remainder of the 6-week study period. All Headspace courses teach mindfulness using various basic techniques, including breathing exercises, body scan, noting (being aware of any emotions that may be arising at the moment), and visualization (visualizing images such as sun shining on the entire body). In addition to the daily, progressive audio instruction, there are occasional short (1-2 min) lecture videos designed to increase the understanding of mindfulness and to encourage its integration into daily life. Headspace can be set up to send reminders using push notifications.

\section{Measures}

\section{Feasibility}

We assessed the feasibility of the intervention on the criteria outlined by Bowen et al. (2009). Demand was evaluated by calculating the number of women with a positive antenatal PHQ-9 screening score assessed by KPNC clinicians via routine screening and identified from the EHR. Acceptability was assessed through responses in a semi-structured interview. Women were asked open-ended questions about their experience with the study and with Headspace, recommended changes to the study procedures, and perceived need for additional health system support for pregnant women. Participants' responses were written down as close to verbatim as possible by the interviewer. Implementation was evaluated by measuring retention, which was calculated as the proportion of enrolled participants who completed both the baseline and post-intervention surveys. Practicality was operationalized as adherence to the mindfulness program. Date, duration, and name of each meditation were ascertained from Headspace for each participant using the study-specific log-in ID, enabling us to calculate adherence. Integration was assessed by asking participants during the semi-structured interview about perceived effects/benefits of practicing mindfulness and intention to continue using the Headspace program. Expansion focuses on the scalability of the intervention. Preliminary efficacy was evaluated by assessing change in patient-reported outcomes.

\section{Depression}

The 8-item PHQ-8 (Kroenke et al. 2009) depression screener is a validated instrument adapted from the PHQ- 9 and was used to assess current depression symptoms at recruitment and follow-up. The PHQ-8 excludes the question regarding suicidal thoughts. PHQ-8 scores range from 0 to 24. A score of 1-4 suggests minimal depression, 5-9 mild depression, 10-
14 moderate depression, $15-19$ moderately severe depression, and 20-24 severe depression. The PHQ- 8 has been validated and reported to have an alpha coefficient of 0.82 (Pressler et al. 2011).

\section{Stress}

The 10-item Perceived Stress Scale (PSS-10) (Cohen and Williamson 1988) assesses to what degree a respondent perceives situations in his or her life in the past month as stressful through a 5 -point Likert scale $(0=$ "Never" to $4=$ "Very Often"). The scores are summed to give a total score ranging from 0 to 40 . The PSS-10 has been demonstrated as having high internal consistency ( $\alpha=0.85)$ (Smith et al. 2014).

\section{Sleep Quality}

The 19-item Pittsburgh Sleep Quality Index (PSQI) (Buysse et al. 1989) asks about sleep quality during the past month, including questions on sleep duration, sleep disturbance, and use of sleep-inducing medications. A global score, ranging from 0 to 21, is calculated using seven components of sleep. Higher scores indicate poorer sleep quality. In a study of pregnant women, the PSQI was validated at two time points: 15-23 weeks, and 29-39 weeks of gestation. Alpha coefficients were 0.70 and 0.76 , respectively (Skouteris et al. 2009).

\section{Mindfulness}

The 24-item Five Facet Mindfulness Questionnaire short form (FFMQ-SF) (Bohlmeijer et al. 2011) uses a 5-point Likert scale to measure five factors representing elements of mindfulness: observing, describing, acting with awareness, nonjudging of inner experience, and nonreactivity to inner experience. Responses vary between 1 ("never or very rarely true") and 5 ("very often or always true"). Higher scores indicate greater mindfulness. The FFMQ-SF has good reliability with alpha coefficients ranging from 0.80 (nonreactivity) to 0.90 (acting with awareness, describing, nonjudging) (Pelham et al. 2019).

\section{Data Analyses}

Baseline differences between completers and non-completers were assessed using ANOVA for continuous variables and Fisher's exact test for categorical variables. Mean and standard deviations were calculated for all participant-reported outcome measures. Change in score and $p$ values were derived using paired $t$ tests. Cohen's $d$ effect sizes were calculated by dividing change in score by the standard deviation of change in score. All analyses were conducted using SAS v. 9.4 (Cary, $\mathrm{NC})$. 
Data from semi-structured interviews were uploaded into NVivo qualitative data analysis software (QSR International Pty Ltd., version 12, 2018.). Inductive thematic analysis was used to identify and develop codes on themes related to mindfulness benefits, the interface experience, experience with the study, and suggested changes. Two primary coders (MM and LN) coded each interview. A third coder (EK) reviewed all coded transcripts to ensure accuracy of codes.

\section{Results}

\section{Baseline Characteristics}

Patients who completed the study were more likely to be White $(65 \%)$ and less likely to be Black $(10 \%)$ or multiracial $(5 \%)$, more likely to have at least a college degree (75\%), less likely to be primigravid (65\%), and more likely to be married (80\%) compared to the women who did not complete the study (Table 1). Women who completed the study were slightly older than those who did not complete the study. Noncompleters had higher baseline PHQ-8 scores (15.7) compared with completers (13.5) and were slightly farther along in pregnancy (17.7 weeks of gestation) compared with completers (16.3 weeks of gestation). However, these differences were not statistically significant.

\section{Demand}

The number of women with moderate depression symptoms was high. Over 500 women in seven clinics had a PHQ-9 score of 10-19, among approximately 6400 pregnant women who were screened during the study period.

\section{Acceptability}

Fifteen participants $(75 \%)$ completed a brief, postintervention telephone interview. Ten participants (67\%) who responded reported that they were either "very" or "extremely satisfied" with the Headspace app. Overall, participants felt it was easy to use, appreciated the convenience of the app (e.g., able to use at any time and any location), and liked the variety of meditation packs available. Some participants disliked the meditation facilitator's voice and reported they would have liked to have different voice options.

\section{Implementation}

The study had a high retention rate. Of the 27 participants, 20 (74\%) completed the baseline and follow-up survey.

\section{Practicality}

Among the women who completed the study, 19 (95\%) used Headspace at least once. Of the 19 participants, 11 (58\%) practiced meditation $\geq 50 \%$ of the days during the 6week study period, and five (26\%) practiced $\geq 70 \%$ of the days. In addition, $53 \%$ of participants used Headspace at least once during the month after the intervention period ended. In addition to the Headspace "Basics" packs, the most frequently used packs included "Pregnancy," a 30day program that uses a visualization technique to cultivate connection with the newborn, as well as to prepare for change in life; "Managing Anxiety," a 30-day program that uses "noting" and "body scan" techniques to manage anxiety; and other programs that help with sleep. Study staff called 18 women $(67 \%)$ at least once during the study to remind them to engage in the intervention due to failure to complete at least three Headspace sessions in the previous week.

\section{Integration}

The most commonly reported perceived benefits of the meditation program were decreased anxiety, increased calmness, improved sleep, and being able to set aside time for themselves and to quiet their minds. Most felt that the first trimester was the best time to start the meditation program, preferably as early as possible after learning about the pregnancy. All participants interviewed said they were planning to continue using Headspace after the end of the study.

\section{Expansion}

The intervention used a commercially available mindfulness app. This makes the intervention scalable and widely implementable in a wide range of health care settings as it does not require training of facilitators or fidelity control.

\section{Preliminary Efficacy}

Compared to baseline, at the 6-week post-intervention followup, participants experienced significant improvements in depression symptoms $(-6.0, p \leq .01)$, perceived stress $(-5.6, p \leq$ $.01)$, and sleep quality $(-1.9, p \leq .05$; with these findings indicating less sleep disturbance) (Table 2). Participants also achieved greater levels of mindfulness in four of five mindfulness domains (observing: $+1.4, p \leq .05$; describing: $+2.1, p$ $\leq .01$; acting with awareness: $+3.2, p \leq .01$; nonreactivity: $+2.1, p \leq .05$ ) and significant improvements in overall mindfulness $(+10.6, p \leq .01)$. 
Table 1 Baseline demographic and clinical characteristics of participants

\begin{tabular}{|c|c|c|c|}
\hline Baseline characteristics & $\begin{array}{l}\text { Total } \\
n=27\end{array}$ & $\begin{array}{l}\text { Completed } \\
n=20\end{array}$ & $\begin{array}{l}\text { Not completed } \\
n=7\end{array}$ \\
\hline Gestation (weeks), mean (range) & $16.7(9-30)$ & $16.3(9-29)$ & $17.7(13-30)$ \\
\hline \multicolumn{4}{|l|}{ Parity, $n(\%)$} \\
\hline 0 & $19(70.4)$ & $13(65.0)$ & $6(85.7)$ \\
\hline $1+$ & $8(29.6)$ & $7(35.0)$ & $1(14.3)$ \\
\hline \multicolumn{4}{|l|}{ Depression diagnosis ${ }^{\mathrm{a}}, n(\%)$} \\
\hline Pre-pregnancy & $4(14.8)$ & $2(10.0)$ & $2(28.6)$ \\
\hline Pregnancy & $3(11.1)$ & $2(10.0)$ & $1(14.3)$ \\
\hline Both & $7(25.9)$ & $6(30.0)$ & $1(14.3)$ \\
\hline Neither & $13(48.2)$ & $10(50.0)$ & $3(42.9)$ \\
\hline Baseline PHQ-8 score, mean (range) & $14.0(10-22)$ & $13.5(10-21)$ & $15.7(11-22)$ \\
\hline Age, mean (range) & $31.0(19-39)$ & $31.4(19-39)$ & $29.9(20-39)$ \\
\hline \multicolumn{4}{|l|}{ Race/ethnicity, $n(\%)$} \\
\hline White & $16(59.3)$ & $13(65.0)$ & $3(42.9)$ \\
\hline Black or African American & $3(11.1)$ & $2(10.0)$ & $1(14.3)$ \\
\hline Asian & $1(3.7)$ & $1(5.0)$ & $0(0.0)$ \\
\hline Hispanic & $4(14.8)$ & $3(15.0)$ & $1(14.3)$ \\
\hline Multiracial & $3(11.1)$ & $1(5.0)$ & $2(28.6)$ \\
\hline \multicolumn{4}{|l|}{ Education, $n(\%)$} \\
\hline High school or less & $3(11.1)$ & $2(10.0)$ & $1(14.3)$ \\
\hline Some college/vocational school & $6(22.2)$ & $3(15.0)$ & $3(42.9)$ \\
\hline College graduate & $18(66.7)$ & $15(75.0)$ & $3(42.9)$ \\
\hline \multicolumn{4}{|l|}{ Household income, $n(\%)$} \\
\hline$<\$ 100,000$ & $15(55.6)$ & $11(55.0)$ & $4(57.1)$ \\
\hline$\geq \$ 100,000$ & $12(44.4)$ & $9(45.0)$ & $3(42.9)$ \\
\hline \multicolumn{4}{|l|}{ Medicaid status, $n(\%)$} \\
\hline Yes & $5(20.0)$ & $4(22.2)$ & $1(14.3)$ \\
\hline No & $20(80.0)$ & $14(77.8)$ & $6(85.7)$ \\
\hline \multicolumn{4}{|l|}{ Marital status, $n(\%)$} \\
\hline Married & $20(74.1)$ & $16(80.0)$ & $4(57.1)$ \\
\hline Never married & $7(25.9)$ & $4(20.0)$ & $3(42.9)$ \\
\hline \multicolumn{4}{|c|}{ Primary device used for mindfulness program, $n(\%)$} \\
\hline Android & $6(26.1)$ & $6(31.6)$ & $0(0.0)$ \\
\hline iOS & $15(65.2)$ & $13(68.4)$ & $2(50.0)$ \\
\hline Desktop & $2(8.7)$ & $0(0.0)$ & $2(50.0)$ \\
\hline
\end{tabular}

${ }^{a}$ Depression diagnosis codes were ascertained from the electronic health records for the period of 1 year prior to their pregnancy through study recruitment. Participants were categorized as having pre-pregnancy depression, antenatal depression, both, or neither based on the dates of the diagnosis and dates of current pregnancy

\section{Discussion}

Findings from this study using criteria from Bowen et al. (2009) suggest feasibility and acceptability of a self-paced mHealth mindfulness intervention for pregnant women with moderate-to-moderately-severe levels of depression. Participants appreciated the convenience of the intervention, and many learned mindfulness skills using mHealth technology. Although this study was not designed as an efficacy trial, the preliminary results of self-reported outcomes as well as semi-structured interviews suggest improvement in depression symptoms, stress, sleep, and mindfulness. These findings are important given the potential impact that this mHealth mindfulness intervention may have on reducing health care costs. Further, effective mHealth interventions for antenatal depression may help address the severe shortage in mental health care resources, which has been exasperated during the COVID-19 pandemic. The methods used provide a practical strategy for conducting a larger, fully powered trial, and information gained from this feasibility study will provide 
Table 2 Baseline and post-intervention outcome measures

\begin{tabular}{|c|c|c|c|c|c|c|}
\hline Outcome & Possible score range & $\begin{array}{l}\text { Baseline } \\
\text { Mean (SD) }\end{array}$ & $\begin{array}{l}\text { Post-intervention } \\
\text { Mean (SD) }\end{array}$ & $\begin{array}{l}\text { Change } \\
\text { Mean (SD) }\end{array}$ & Cohen's $d$ & $p$ value \\
\hline Patient Health Questionnaire (PHQ-8) & $0-24$ & $13.5(3.5)$ & $7.5(4.4)$ & $-6.0(5.5)$ & -1.1 & $<.001$ \\
\hline Perceived Stress Scale (PSS-10) & $10-40$ & $24.5(5.7)$ & $18.9(6.4)$ & $-5.6(7.3)$ & -0.8 & .0027 \\
\hline Pittsburgh Sleep Quality Index (PSQI) & $0-21$ & $10.9(3.8)$ & $9.1(3.9)$ & $-1.9(3.3)$ & -0.6 & .0224 \\
\hline \multicolumn{7}{|c|}{ Five Facet Mindfulness Questionnaire (FFMQ) } \\
\hline Observing & $4-20$ & $13(3.8)$ & $14.1(3.5)$ & $1.4(2.8)$ & 0.5 & .0467 \\
\hline Describing & $5-25$ & $17.4(3.7)$ & $19.5(3.7)$ & $2.1(2.3)$ & 0.9 & $<.001$ \\
\hline Acting with awareness & $5-25$ & $14.1(2.9)$ & $17.3(3.8)$ & $3.2(3.4)$ & 0.9 & $<.001$ \\
\hline Nonjudging of inner experience & $5-25$ & $13.2(3.5)$ & $14.9(4.1)$ & $1.8(4.5)$ & 0.4 & .0987 \\
\hline Nonreactivity & $5-25$ & $12.7(3.9)$ & $14.8(3.2)$ & $2.1(3.5)$ & 0.6 & .0184 \\
\hline Total & $24-120$ & $70.3(11.3)$ & $80.6(13.1)$ & $10.6(11.7)$ & 0.9 & .001 \\
\hline
\end{tabular}

important insights to increase the likelihood of a successful future study.

Mindfulness is a mental state achieved by focusing one's awareness on the present moment with an attitude of nonjudgment (Kabat-Zinn 1990). The theoretical basis for mindfulness derives from a model based on studies of the influence of mindfulness on brain regions involved in learning and memory processes, emotion regulation, self-referential processing, and perspective taking (Holzel et al. 2011a, b). A recent meta-analysis of nine studies targeting depressed adults ( $75 \%$ women) reported that those who received the mindfulness-based intervention had a significantly reduced risk of depression relapse (5-year follow-up period) compared with those in the control group (hazard ratio, 0.69; 95\% CI, 0.58-0.82) (Kuyken et al. 2016). Similar to our findings, a recent meta-analysis of eight randomized controlled trials of pregnant women concluded that women in the in-person mindfulness arm experienced significant reductions in depression, anxiety, and other quality of life measures with small to medium effect sizes (Lever Taylor et al. 2016). Thus, mindfulness programs have promise as non-pharmacological interventions to help women suffering from depression.

The accessibility challenges of in-person mindfulness training and the recent COVID-19 pandemic underscore the need for accessible and robust resources for mental health, with high priority in an extremely vulnerable populationpregnant women. A recent survey found that the COVID-19 pandemic has had a significant impact on the mental health of pregnant and postpartum women with over a third of women reporting significant depression symptoms (American Psychiatric Association 2020). This increase in the number of women with antenatal depression symptoms is coupled with a surge in demand for mental health care. The mental health care system nationwide was suffering from a shortage of providers prior to the COVID-19 pandemic (Andrilla et al. 2018; Kaiser Family Foundation n.d.; Walker et al. 2015). Now, months into the COVID-19 outbreak, mental health care providers are in extreme shortage (Li 2020; Panchal et al. 2020). The pandemic has also forced health care services, including mental health care, to be delivered remotely (Bojdani et al. 2020). Thus, there is an urgent and critical need for the identification of remotely-delivered, scalable, and effective interventions.

There have been a limited number of studies that have used the Internet to deliver mindfulness interventions to pregnant women. A study conducted in China that targeted pregnant women with mild or moderate symptoms of depression and anxiety found that an Internet-based mindfulness intervention improved depression and anxiety (Yang et al. 2019), while a UK-based study did not find a significant benefit of a Webbased intervention, mainly due to high attrition rates ( 22 out of 107 in intervention arm completed the study) (Krusche et al. 2018). There are also a few other studies using Web-based mindfulness interventions that are currently underway, though the results are not yet available (Cohen and Dimidjian 2018; Hulsbosch et al. 2020). The present study differs from these studies because we used a self-paced, mobile-based intervention that does not require logging into sessions at specified time. To our knowledge, there has not been any mHealth mindfulness study targeting pregnant women with depressive symptoms. Other studies that used Headspace have demonstrated a reduction in stress and improvement in well-being in general populations (Champion et al. 2018; Economides et al. 2018). Further, research has demonstrated a reduction in distress and improvement in quality of life for cancer patients using the Headspace intervention (Kubo et al. 2018, 2019, 2020).

In the present study, the participants appreciated the intervention because of the ease of using the program and the short dosage (10-20 min a day). They also welcomed the fact that they could use their mobile phone or computer to engage in the intervention without having to leave home or work to attend in-person classes. Most women we reached had access to the technology to receive the mHealth intervention, and 
among those who enrolled, very few participants had trouble downloading or using the app. Nearly a quarter of our sample received Medicaid benefits at study baseline, highlighting the potential for mHealth mindfulness-based interventions to reach women of low socioeconomic status with moderate-tomoderately-severe antenatal depression symptoms. This is important as these women often do not have access to more traditional treatment options (e.g., psychotherapy) for antenatal depression. Mobile devices are increasingly used by all segments of the population, with approximately $90 \%$ of Americans of reproductive age owning a smartphone (PEW Internet and American Life Project 2017). If this mHealth mindfulness intervention is found to be effective, it may serve as a low-cost strategy to help a large number of women suffering from antenatal depression with limited access to care. Lastly, half our sample experienced moderate-to-moderatelysevere depression symptoms that did not reach the threshold for a clinical diagnosis. The US Preventive Services Task Force's recent recommendation to refer all women at increased risk of perinatal depression to counseling services will be met with the patient-level barriers (e.g. costs, time) as well as challenges from the health care system, which is already suffering from a shortage of mental health care providers (Avalos et al. 2019; Felder 2019; Freeman 2019; O'Connor et al. 2019; Wisner et al. 2019). Findings from this study suggest the feasibility of an mHealth mindfulness-based intervention for women at increased risk of perinatal depression if future studies provide evidence of efficacy of mHealth mindfulness-based interventions in this population.

Although this was a feasibility study that was not designed to be an efficacy study, we observed a reduction in depression scores after the intervention. The decrease in mean post-PHQ8 scores to below the threshold for a positive screen (PHQ-8 score of 10 or greater) accompanied by a nearly $50 \%$ reduction in symptoms highlight the clinical significance of the improvement in depression symptom burden (Kroenke et al. 2009). Although the pre- and post-perceived stress scores both correspond to a moderate level of stress (PSS scores between 14 and 26), we documented a reduction in symptoms of perceived stress of nearly a quarter (24\%) (Cohen et al. 1983). A $20 \%$ reduction in the sleep quality score was noted, although the mean post-score did not fall below the threshold of 5, and scores were still indicative of poor sleep quality (Akman et al. 2015).

A few lessons were learned from this feasibility study. First, the recruitment rate was higher when clinicians referred patients to the study compared to when study staff reached out about the study. This underscores the importance of engaging clinicians when conducting intervention studies to maximize recruitment rates. Second, we tracked participants' app usage and reached out when there was an extended period of inactivity (i.e., $<3$ sessions in the past week). This helped remind some participants to get back on track with the app. Using the reminder features and push notification built into the app may also be useful as a reminder given the proportion of women our study team reached out to with reminder calls. Additionally, automatic tracking of progress can be used as a source of motivation to continue using the program. Such interactive features of a mobile app can increase its adherence and effectiveness, and ease of using the app can facilitate widespread, efficient implementation (Hebden et al. 2012).

\section{Limitations and Future Research Directions}

The present study has several limitations. First, since it is a small single-arm feasibility study, we cannot draw conclusions regarding the efficacy of the intervention on participant-reported outcomes or assess dose-response relationships. However, the purpose of the study was to determine feasibility and acceptability, which we were able to demonstrate. The preliminary efficacy results on patient-reported outcomes were promising and provided tentative effect size estimates to conduct an empirically informed power analysis for a larger randomized control trial. Second, because we required the use of technology as part of the intervention, the study findings may not be generalizable to the general population due to the "digital divide." However, as noted, the majority of the women we attempted to recruit owned mobile devices or computers with an Internet connection, and a quarter of our sample were on Medicaid. The main reasons reported for declining to participate were lack of time or interest, not lack of access to technology. Third, as in most intervention studies, the women who completed and those who dropped out were slightly different from each other, potentially affecting the generalizability of the results. However, unlike most previous studies of mindfulness interventions which tend to be predominantly among Whites, this study enrolled women of minority backgrounds as well as low-income women. Future studies focusing on these vulnerable populations would help reduce health disparities with regard to access to mental health care. Fourth, non-English speakers were ineligible because the intervention is only offered in English. Future mindfulnessbased studies should incorporate other languages given the high burden of depression among minority populations (Mukherjee et al. 2016).

Given the promising results from this feasibility study, larger-scale randomized trials are needed to establish the efficacy and durability of mHealth mindfulness interventions in this population. Pragmatic trials of this type of intervention within large health care systems will help provide evidence regarding the effectiveness of implementing a low-cost, technology-based program for pregnant women suffering from moderate-to-moderately-severe depression symptoms, with a goal of improving the health of both mothers and the offspring. 
Author Contribution AK conceptualized and designed the study, obtained funding, drafted parts of the initial manuscript, and reviewed and revised the final manuscript. SA extracted the data, created the database for analysis, analyzed the data, drafted parts of the initial manuscript, and reviewed and revised the final manuscript. EMK prepared the study materials, managed the study recruitment, collected the participant data, conducted the qualitative data analyses, drafted parts of the initial manuscript, and reviewed and revised the final manuscript. LN prepared the study materials, managed the study recruitment, collected the participant data, conducted the qualitative data analyses, and reviewed and revised the final manuscript. CPQ advised on the statistical analyses and interpretation of the results and reviewed and revised the final manuscript. MKM prepared the study materials, managed the study recruitment, collected the participant data, conducted the qualitative data analyses, and reviewed and revised the final manuscript. LAA conceptualized and designed the study, obtained funding, drafted parts of the initial manuscript, and reviewed and revised the final manuscript.

Funding This study was funded by Kaiser Permanente Northern California Community Benefits and National Institutes of Health (grant K01MH103444 to Dr. Avalos and K07 CA166143 to Dr. Kubo).

\section{Declarations}

Ethics Approval and Consent to Participate All procedures performed in studies involving human participants were in accordance with the ethical standards of the institutional and/or national research committee (Kaiser Permanente Northern California Institutional Review Board (\#1279553)) and with the 1964 Helsinki Declaration and its later amendments or comparable ethical standards. Informed consent was obtained from all individual participants included in the study.

Conflict of Interest The authors declare no competing interests.

\section{References}

Akman, T., Yavuzsen, T., Sevgen, Z., Ellidokuz, H., \& Yilmaz, A. U. (2015). Evaluation of sleep disorders in cancer patients based on Pittsburgh Sleep Quality Index. European Journal of Cancer Care, 24(4), 553-559. https://doi.org/10.1111/ecc.12296.

American Psychiatric Association. (2020). COVID-19 impacting mental well-being: Americans feeling anxious, especially for loved ones; older adults are less anxious. https://www.psychiatry.org/ newsroom/news-releases/new-poll-covid-19-impacting-mentalwell-being-americans-feeling-anxious-especially-for-loved-onesolder-adults-are-less-anxious.

Andrilla, C. H. A., Patterson, D. G., Garberson, L. A., Coulthard, C., \& Larson, E. H. (2018). Geographic variation in the supply of selected behavioral health providers. American Journal of Preventive Medicine, 54(6 Suppl 3), S199-s207. https://doi.org/10.1016/j. amepre.2018.01.004.

Avalos, L. A., Chen, H., \& Li, D. K. (2015). Antidepressant medication use, depression, and the risk of preeclampsia. CNS Spectrums, 20(1), 39-47. https://doi.org/10.1017/s1092852915000024.

Avalos, L. A., Flanagan, T., \& Li, D. K. (2019). Preventing perinatal depression to improve maternal and child health-A health care imperative. JAMA Pediatrics, 173(4), 313-314. https://doi.org/10. 1001/jamapediatrics.2018.5491.

Badon, S. E., Hedderson, M. M., Hyde, R. J., Quesenberry, C. P., \& Avalos, L. A. (2019). Pre- and early pregnancy onset depression and subsequent rate of gestational weight gain. Journal of
Women's Health, 28(9), 1237-1245. https://doi.org/10.1089/jwh. 2018.7497.

Bohlmeijer, E., ten Klooster, P. M., Fledderus, M., Veehof, M., \& Baer, R. (2011). Psychometric properties of the five facet mindfulness questionnaire in depressed adults and development of a short form. Assessment, 18(3), 308-320. https://doi.org/10.1177/ 1073191111408231.

Bojdani, E., Rajagopalan, A., Chen, A., Gearin, P., Olcott, W., Shankar, V., Cloutier, A., Solomon, H., Naqvi, N. Z., Batty, N., Festin, F. E. D., Tahera, D., Chang, G., \& DeLisi, L. E. (2020). COVID-19 pandemic: Impact on psychiatric care in the United States. Psychiatry Research, 289, 113069. https://doi.org/10.1016/j. psychres.2020.113069.

Bowen, D. J., Kreuter, M., Spring, B., Cofta-Woerpel, L., Linnan, L., Weiner, D., Bakken, S., Kaplan, C. P., Squiers, L., \& Fabrizio, C. (2009). How we design feasibility studies. American Journal of Preventive Medicine, 36(5), 452-457. https://doi.org/10.1016/j. amepre.2009.02.002.

Bruggeman-Everts, F. Z., Wolvers, M. D. J., van de Schoot, R., Vollenbroek-Hutten, M. M. R., \& Van der Lee, M. L. (2017). Effectiveness of two web-based interventions for chronic cancerrelated fatigue compared to an active control condition: Results of the "Fitter na kanker" randomized controlled trial. Journal of Medical Internet Research, 19(10), e336. https://doi.org/10.2196/ jmir.7180.

Buysse, D. J., Reynolds, C. F., Monk, T. H., Berman, S. R., \& Kupfer, D. J. (1989). The Pittsburgh Sleep Quality Index: A new instrument for psychiatric practice and research. Psychiatry Research, 28(2), 193-213.

Champion, L., Economides, M., \& Chandler, C. (2018). The efficacy of a brief app-based mindfulness intervention on psychosocial outcomes in healthy adults: A pilot randomised controlled trial. PLoS One, 13(12), e0209482. https://doi.org/10.1371/journal.pone.0209482.

Cohen, L., \& Dimidjian, S. (2018). Preventing depressive relapse in pregnant women with recurrent depression [Identifier NCT03623620]. National Library of Medicine (U.S.). https:// clinicaltrials.gov/ct2/show/NCT03623620.

Cohen, C. K., \& Williamson, G. (1988). Perceived stress in a probability sample of the United States. In Social Psychology of Health. Sage.

Cohen, S., Kamarck, T., \& Mermelstein, R. (1983). A global measure of perceived stress. Journal of Health and Social Behavior, 385-396. https://doi.org/10.2307/2136404.

Compen, F., Bisseling, E., Schellekens, M., Donders, R., Carlson, L., van der Lee, M., \& Speckens, A. (2018). Face-to-face and Internet-based mindfulness-based cognitive therapy compared with treatment as usual in reducing psychological distress in patients with cancer: A multicenter randomized controlled trial. Journal of Clinical Oncology, 36(23), 2413-2421. https://doi.org/10.1200/JCO.2017. 76.5669 .

Creswell, J. D. (2017). Mindfulness interventions. Annual Review of Psychology, 68, 491-516. https://doi.org/10.1146/annurev-psych042716-051139.

Davenport, M. H., Meyer, S., Meah, V. L., Strynadka, M. C., \& Khurana, R. (2020). Moms are not OK: COVID-19 and maternal mental health. Frontiers in Global Women's Health, 1, 1. https://doi.org/ 10.3389/fgwh.2020.00001.

Dimidjian, S., \& Goodman, S. (2009). Nonpharmacologic intervention and prevention strategies for depression during pregnancy and the postpartum. Clinical Obstetrics and Gynecology, 52(3), 498-515. https://doi.org/10.1097/GRF.0b013e3181b52da6.

Dimidjian, S., \& Goodman, S. H. (2014). Preferences and attitudes toward approaches to depression relapse/recurrence prevention among pregnant women. Behaviour Research and Therapy, 54, 7-11. https://doi.org/10.1016/j.brat.2013.11.008.

Dimidjian, S., Goodman, S. H., Felder, J. N., Gallop, R., Brown, A. P., \& Beck, A. (2016). Staying well during pregnancy and the postpartum: 
A pilot randomized trial of mindfulness-based cognitive therapy for the prevention of depressive relapse/recurrence. Journal of Consulting and Clinical Psychology, 84(2), 134-145. https://doi. org/10.1037/ccp0000068.

Economides, M., Martman, J., Bell, M. J., \& Sanderson, B. (2018). Improvements in stress, affect, and irritability following brief use of a mindfulness-based smartphone app: a randomized controlled trial. Mindfulness, 9(5), 1584-1593. https://doi.org/10.1007/ s12671-018-0905-4.

Economides, M., Ranta, K., Nazander, A., Hilgert, O., Goldin, P. R., Raevuori, A., \& Forman-Hoffman, V. (2019). Long-term outcomes of a therapist-supported, smartphone-based intervention for elevated symptoms of depression and anxiety: Quasiexperimental, prepostintervention study. Journal of Medical Internet Research mHealth and uHealth, 7(8), e14284. https://doi.org/10.2196/14284.

Eyles, C., Leydon, G. M., Hoffman, C. J., Copson, E. R., Prescott, P., Chorozoglou, M., \& Lewith, G. (2015). Mindfulness for the selfmanagement of fatigue, anxiety, and depression in women with metastatic breast cancer: A mixed methods feasibility study [Research Support, Non-U.S. Gov't]. Integrative Cancer Therapies, 14(1), 42-56. https://doi.org/10.1177/ 1534735414546567

Felder, J. N. (2019). Implementing the USPSTF recommendations on prevention of perinatal depression - Opportunities and challenges. JAMA Internal Medicine, 179(4), 467-468. https://doi.org/10.1001/ jamainternmed.2018.7729.

Freeman, M. P. (2019). Perinatal depression: Recommendations for prevention and the challenges of implementation. JAMA, 321(6), 550 552. https://doi.org/10.1001/jama.2018.21247.

Gavin, N. I., Gaynes, B. N., Lohr, K. N., Meltzer-Brody, S., Gartlehner, G., \& Swinson, T. (2005). Perinatal depression: A systematic review of prevalence and incidence. Obstetrics \& Gynecology, 106(5 Part 1), 1071-1083. https://doi.org/10.1097/01.AOG.0000183597. 31630.db.

Gaynes, B. N., Gavin, N., Meltzer-Brody, S., Lohr, K. N., Swinson, T., Gartlehner, G., Brody, S., \& Miller, W. C. (2005). Perinatal depression: Prevalence, screening accuracy, and screening outcomes. Evidence Report/Technology Assessment (Summary), (119), 1-8 http://www.ncbi.nlm.nih.gov/entrez/query.fcgi?cmd= Retrieve $\& \mathrm{db}=\mathrm{PubMed} \& d o p t=C i t a t i o n \& l i s t \_u i d s=15760246$.

Goodman, J. H. (2009). Women's attitudes, preferences, and perceived barriers to treatment for perinatal depression. Birth, 36(1), 60-69. https://doi.org/10.1111/j.1523-536X.2008.00296.x.

Gordon, N. (2012). A comparison of sociodemographic and health characteristics of the Kaiser Permanente Northern California membership derived from two data sources: The 2008 Member Health Survey and the 2007 California Health Interview Survey.

Gordon, N. (2015). Similarity of the adult Kaiser Permanente membership in Northern California to the insured and general population in Northern California: Statistics from the 2011-12 California Health Interview Survey. https://divisionofresearch.kaiserpermanente.org/ projects/memberhealthsurvey/SiteCollectionDocuments/chis_non_ kp 2011.pdf.

Grigoriadis, S., VonderPorten, E. H., Mamisashvili, L., Tomlinson, G., Dennis, C. L., Koren, G., Steiner, M., Mousmanis, P., Cheung, A., Radford, K., Martinovic, J., \& Ross, L. E. (2013). The impact of maternal depression during pregnancy on perinatal outcomes: A systematic review and meta-analysis. Journal of Clinical Psychiatry, 74(4), e321-e341. https://doi.org/10.4088/JCP. $12 \mathrm{r} 07968$.

Guardino, C. M., Dunkel Schetter, C., Bower, J. E., Lu, M. C., \& Smalley, S. L. (2014). Randomised controlled pilot trial of mindfulness training for stress reduction during pregnancy. Psychology \& Health, 29(3), 334-349. https://doi.org/10.1080/08870446.2013. 852670 .
Hebden, L., Cook, A., van der Ploeg, H. P., \& Allman-Farinelli, M. (2012). Development of smartphone applications for nutrition and physical activity behavior change. JMIR Research Protocols, 1(2), e9. https://doi.org/10.2196/resprot.2205.

Holzel, B. K., Carmody, J., Vangel, M., Congleton, C., Yerramsetti, S. M., Gard, T., \& Lazar, S. W. (2011a). Mindfulness practice leads to increases in regional brain gray matter density. Psychiatry Research, 191(1), 36-43. https://doi.org/10.1016/j.pscychresns.2010.08.006.

Holzel, B. K., Lazar, S. W., Gard, T., Schuman-Olivier, Z., Vago, D. R., \& Ott, U. (2011b). How does mindfulness meditation work? Proposing mechanisms of action from a conceptual and neural perspective. Perspectives on Psychological Science: a Journal of the Association for Psychological Science, 6(6), 537-559. https://doi. org/10.1177/1745691611419671.

Hulsbosch, L. P., Nyklíček, I., Potharst, E. S., Meems, M., Boekhorst, M., \& Pop, V. J. M. (2020). Online mindfulness-based intervention for women with pregnancy distress: Design of a randomized controlled trial. BMC Pregnancy and Childbirth, 20(1), 159. https://doi.org/10. 1186/s12884-020-2843-0.

Kabat-Zinn, J. (1990). Full catastrophe living: Using the wisdom of your body and mind to face stress, pain, and illness. Delacorte.

Kaiser Family Foundation. (n.d.) Mental health care health professional shortage areas (HPSAs). Retrieved Sept 30 from https://www.kff. org/other/state-indicator/mental-health-care-health-professionalshortage-areas-hpsas/?currentTimeframe $=0 \&$ sortModel $=\% 7 \mathrm{~B} \%$ 22colId\%22:\%22Location\%22,\%22sort\%22:\%22asc\%22\%7D.

Khoury, B., Sharma, M., Rush, S. E., \& Fournier, C. (2015). Mindfulness-based stress reduction for healthy individuals: A meta-analysis. Journal of Psychosomatic Research, 78(6), 519-528. https://doi.org/10.1016/j.jpsychores.2015.03.009.

Ko, J. Y., Farr, S. L., Dietz, P. M., \& Robbins, C. L. (2012). Depression and treatment among U.S. pregnant and nonpregnant women of reproductive age, 2005-2009. Journal of Women's Health, 21(8), 830-836. https://doi.org/10.1089/jwh.2011.3466.

Kroenke, K., Spitzer, R. L., \& Williams, J. B. (2001). The PHQ-9: Validity of a brief depression severity measure. Journal of General Internal Medicine, 16(9), 606-613. https://doi.org/10. 1046/j.1525-1497.2001.016009606.x.

Kroenke, K., Strine, T. W., Spitzer, R. L., Williams, J. B., Berry, J. T., \& Mokdad, A. H. (2009). The PHQ-8 as a measure of current depression in the general population. Journal of Affective Disorders, 114(1), 163-173. https://doi.org/10.1016/j.jad.2008.06.026.

Krusche, A., Dymond, M., Murphy, S. E., \& Crane, C. (2018). Mindfulness for pregnancy: A randomised controlled study of online mindfulness during pregnancy. Midwifery, 65, 51-57. https:// doi.org/10.1016/j.midw.2018.07.005.

Kubo, A., Altschuler, A., Kurtovich, E., Hendlish, S., Laurent, C. A., Kolevska, T., Li, Y., \& Avins, A. (2018). A pilot mobile-based mindfulness intervention for cancer patients and their informal caregivers. Mindfulness, 9(6), 1885-1894. https://doi.org/10.1007/ s12671-018-0931-2.

Kubo, A., Kurtovich, E., McGinnis, M., Aghaee, S., Altschuler, A., Quesenberry Jr., C., Kolevska, T., \& Avins, A. L. (2019). A randomized controlled trial of mhealth mindfulness intervention for cancer patients and informal cancer caregivers: A feasibility study within an integrated health care delivery system. Integrative Cancer Therapies, 18, 1534735419850634. https://doi.org/10.1177/ 1534735419850634.

Kubo, A., Kurtovich, E., McGinnis, M., Aghaee, S., Altschuler, A., Quesenberry Jr., C., Kolevska, T., Liu, R., Greyz-Yusupov, N., \& Avins, A. (2020). Pilot pragmatic randomized trial of mHealth mindfulness-based intervention for advanced cancer patients and their informal caregivers. Psycho-Oncology. https://doi.org/10. 1002/pon.5557.

Kuyken, W., Warren, F. C., Taylor, R. S., Whalley, B., Crane, C., Bondolfi, G., Hayes, R., Huijbers, M., Ma, H., Schweizer, S., 
Segal, Z., Speckens, A., Teasdale, J. D., Van Heeringen, K., Williams, M., Byford, S., Byng, R., \& Dalgleish, T. (2016). Efficacy of mindfulness-based cognitive therapy in prevention of depressive relapse: An individual patient data meta-analysis from randomized trials. JAMA Psychiatry, 73(6), 565-574. https://doi. org/10.1001/jamapsychiatry.2016.0076.

Lengacher, C. A., Reich, R. R., Ramesar, S., Alinat, C. B., Moscoso, M., Cousin, L., Marino, V. R., Elias, M. N., Paterson, C. L., Pleasant, M. L., Rodriguez, C. S., Wang, H. L., Kip, K. E., Meng, H., \& Park, J. Y. (2018). Feasibility of the mobile mindfulness-based stress reduction for breast cancer (mMBSR(BC)) program for symptom improvement among breast cancer survivors. Psycho-oncology, 27(2), 524-531. https://doi.org/10.1002/pon.4491.

Lever Taylor, B., Cavanagh, K., \& Strauss, C. (2016). The effectiveness of mindfulness-based interventions in the perinatal period: A systematic review and meta-analysis. PLoS One, 11(5), e0155720. https://doi.org/10.1371/journal.pone.0155720.

Li, L. (2020). Challenges and priorities in responding to COVID-19 in inpatient psychiatry. Psychiatric Services, 71(6), 624-626. https:// doi.org/10.1176/appi.ps.202000166.

Mukherjee, S., Trepka, M. J., Pierre-Victor, D., Bahelah, R., \& Avent, T. (2016). Racial/ethnic disparities in antenatal depression in the United States: A systematic review. Maternal and Child Health Journal, 20(9), 1780-1797. https://doi.org/10.1007/s10995-0161989-x.

O’Connor, E., Senger, C. A., Henninger, M. L., Coppola, E., \& Gaynes, B. N. (2019). Interventions to prevent perinatal depression: Evidence report and systematic review for the US Preventive Services Task Force. JAMA, 321(6), 588-601. https://doi.org/10. 1001/jama.2018.20865.

Pagnini, F., \& Philips, D. (2015). Being mindful about mindfulness. Lancet Psychiatry, 2(4), 288-289. https://doi.org/10.1016/S22150366(15)00041-3.

Panchal, N., Kamal, R., Orgera, K., Cox, C., Garfield, R., Hamel, L., Muñana, C., \& Chidambaram, P. (2020). The implications of COVID-19 for mental health and substance use [issue brief]. https://www.kff.org/coronavirus-covid-19/issue-brief/theimplications-of-covid-19-for-mental-health-and-substance-use/.

Pelham III, W. E., Gonzalez, O., Metcalf, S. A., Whicker, C. L., Scherer, E. A., Witkiewitz, K., Marsch, L. A., \& Mackinnon, D. P. (2019). Item response theory analysis of the five facet mindfulness questionnaire and its short forms. Mindfulness, 10(8), 1615-1628.

PEW Internet and American Life Project. (2017). Who owns cellphones. http://www.pewinternet.org/fact-sheet/mobile/.

Pressler, S. J., Subramanian, U., Perkins, S. M., Gradus-Pizlo, I., Kareken, D., Kim, J., Ding, Y., Sauvé, M. J., \& Sloan, R. (2011). Measuring depressive symptoms in heart failure: Validity and reliability of the Patient Health Questionnaire-8. American Journal of Critical Care, 20(2), 146-152.

Ritvo, P., Daskalakis, Z. J., Tomlinson, G., Ravindran, A., Linklater, R., Kirk Chang, M., Knyahnytska, Y., Lee, J., Alavi, N., Bai, S., Harber, L., Jain, T., \& Katz, J. (2019). An online mindfulnessbased cognitive behavioral therapy intervention for youth diagnosed with major depressive disorders: Protocol for a randomized controlled trial. JMIR Research Protocols, 8(7), e11591. https://doi. org/10.2196/11591.

Schapira, M. M., Mackenzie, E. R., Lam, R., Casarett, D., Seluzicki, C. M., Barg, F. K., \& Mao, J. J. (2014). Breast cancer survivors willingness to participate in an acupuncture clinical trial: A qualitative study. Supportive Care in Cancer, 22(5), 1207-1215. https://doi. org/10.1007/s00520-013-2073-3.

Segal, Z. V., Williams, J. M. G., \& Teasdale, J. D. (2002). Mindfulness based cognitive therapy for depression: A new approach to preventing relapse. Guilford Press.
Skouteris, H., Wertheim, E. H., Germano, C., Paxton, S. J., \& Milgrom, J. (2009). Assessing sleep during pregnancy: a study across two time points examining the Pittsburgh Sleep Quality Index and associations with depressive symptoms. Women's Health Issues, 19(1), 4551.

Smith, K. J., Rosenberg, D. L., \& Timothy Haight, G. (2014). An assessment of the psychometric properties of the perceived stress scale-10 (PSS 10) with business and accounting students. Accounting Perspectives, 13(1), 29-59.

Spijkerman, M. P., Pots, W. T., \& Bohlmeijer, E. T. (2016). Effectiveness of online mindfulness-based interventions in improving mental health: A review and meta-analysis of randomised controlled trials. Clinical Psychology Review, 45, 102-114. https://doi.org/10.1016/j. cpr.2016.03.009.

Szegda, K., Markenson, G., Bertone-Johnson, E. R., \& Chasan-Taber, L. (2014). Depression during pregnancy: A risk factor for adverse neonatal outcomes? A critical review of the literature. The Journal of Maternal-Fetal \& Neonatal Medicine, 27(9), 960-967. https://doi. org $/ 10.3109 / 14767058.2013 .845157$.

Vieten, C., \& Astin, J. (2008). Effects of a mindfulness-based intervention during pregnancy on prenatal stress and mood: Results of a pilot study. Archives of Women's Mental Health, 11(1), 67-74. https:// doi.org/10.1007/s00737-008-0214-3.

Wahbeh, H., \& Oken, B. S. (2016). Internet mindfulness meditation intervention for the general public: Pilot randomized controlled trial. JMIR Mental Health, 3(3), e37. https://doi.org/10.2196/mental. 5900.

Wahbeh, H., Lane, J. B., Goodrich, E., Miller, M., \& Oken, B. S. (2014a). One-on-one mindfulness meditation trainings in a research setting. Mindfulness, 5(1), 88-99. https://doi.org/10.1007/s12671-0120155-9.

Wahbeh, H., Svalina, M. N., \& Oken, B. S. (2014b). Group, one-on-one, or Internet? Preferences for mindfulness meditation delivery format and their predictors. Open Medicine Journal, 1, 66. https://doi.org/ 10.2174/1874220301401010066.

Walker, E. R., Cummings, J. R., Hockenberry, J. M., \& Druss, B. G. (2015). Insurance status, use of mental health services, and unmet need for mental health care in the United States. Psychiatric Services, 66(6), 578-584. https://doi.org/10.1176/appi.ps. 201400248.

Wisner, K. L., Miller, E. S., \& Tandon, D. (2019). Attention to prevention-Can we stop perinatal depression before it starts? JAMA Psychiatry, 76(4), 355-356. https://doi.org/10.1001/ jamapsychiatry.2018.4085.

Yang, E., Schamber, E., Meyer, R. M. L., \& Gold, J. I. (2018). Happier healers: Randomized controlled trial of mobile mindfulness for stress management. Journal of Alternative and Complementary Medicine, 24(5), 505-513. https://doi.org/10.1089/acm.2015.0301.

Yang, M., Jia, G., Sun, S., Ye, C., Zhang, R., \& Yu, X. (2019). Effects of an online mindfulness intervention focusing on attention monitoring and acceptance in pregnant women: A randomized controlled trial. Journal of Midwifery \& Women's Health, 64(1), 68-77. https://doi. org/10.1111/jmwh.12944.

Zernicke, K. A., Campbell, T. S., Speca, M., McCabe-Ruff, K., Flowers, S., \& Carlson, L. E. (2014). A randomized wait-list controlled trial of feasibility and efficacy of an online mindfulness-based cancer recovery program: the eTherapy for cancer applying mindfulness trial. Psychosomatic Medicine, 76(4), 257-267. https://doi.org/10. 1097/PSY.0000000000000053.

Publisher's Note Springer Nature remains neutral with regard to jurisdictional claims in published maps and institutional affiliations. 